\title{
UNIQUE LOCALIZATION OF UNKNOWN BOUNDARIES IN A CONDUCTING MEDIUM FROM BOUNDARY MEASUREMENTS
}

\author{
Bruno Canuto ${ }^{1}$
}

\begin{abstract}
We consider the problem of localizing an inaccessible piece $I$ of the boundary of a conducting medium $\Omega$, and a cavity $D$ contained in $\Omega$, from boundary measurements on the accessible part $A$ of $\partial \Omega$. Assuming that $g(t, \sigma)$ is the given thermal flux for $(t, \sigma) \in(0, T) \times A$, and that the corresponding output datum is the temperature $u\left(T_{0}, \sigma\right)$ measured at a given time $T_{0}$ for $\sigma \in A_{\text {out }} \subset A$, we prove that $I$ and $D$ are uniquely localized from knowledge of all possible pairs of input-output data $\left(g, u\left(T_{0}\right)_{\mid A_{\text {out }}}\right)$. The same result holds when a mean value of the temperature is measured over a small interval of time.
\end{abstract}

Mathematics Subject Classification. 35R30.

Received March 3, 2000. Revised February 12, 2001.

\section{INTRODUCTION AND MAIN RESULTS}

In the present paper we are concerned with the study of some problems in thermal imaging. This is a technique used to determine some physical proprieties of a thermic conducting medium via boundary measurements of temperature. More precisely we denote by $\Omega$ the medium, i.e. a bounded and sufficiently smooth domain in $\mathbb{R}^{N}$, $N \geq 2$. Suppose that a piece $I$ of the boundary of $\Omega$ is unknown and inaccessible to direct inspections. On the other hand we have access to the remaining part $A:=(\partial \Omega) \backslash I$ of $\partial \Omega$. Let $g$ be the thermal flux assigned on $(0, T) \times A$, and $u\left(T_{0}\right)_{\mid A_{\text {out }}}$ the corresponding temperature measured at a given time $T_{0}>0$ on a piece $A_{\text {out }}$ of $A$. The goal is to identify the unknown part $I$, by knowing all possible pairs of data $\left(g, u\left(T_{0}\right)_{\mid A_{\text {out }}}\right)$. In a similar problem we might suppose that a cavity $D$, of which neither the form nor the position is known, is contained in $\Omega$ (i.e. $D$ is a domain contained in $\Omega$ ), and the whole boundary of $\Omega$ is known and accessible to measurements. In this case the goal is to identify the cavity $D$ via the same previous data. In fact we are concerned with the problem in which one tries to identify both a piece $I$ of the boundary of $\Omega$ and a cavity $D$ in its interior from all pairs of data $\left(g, u\left(T_{0}\right)_{\mid A_{\text {out }}}\right)$. This problem can occur in nondestructive tests of materials, for example in detecting the corrosion parts of an aircraft which are not accessible to direct inspections. In this case $I$ and $D$ represent the damaged and inaccessible parts of the aircraft, and $u\left(T_{0}\right)_{\mid A_{\text {out }}}$ the measurements of temperature that one disposes to attempt to recover $I$ and $D$ (see Bryan and Caudill [1], and their references).

We denote by $u(t, x)$ the temperature at the time $t$ and at the point $x \in \Omega \backslash \bar{D}, u_{0}$ the initial temperature in $\Omega \backslash \bar{D}, \varphi, \psi$, and $g$ the flux on $(0, T) \times \partial D,(0, T) \times I$, and $(0, T) \times A$ respectively, and $\kappa(x)$ the anisotropic

Keywords and phrases: Inverse boundary value problems, cavities, corrosion, uniqueness.

1 Laboratoire de Mathématiques Appliquées, UMR 7641, Université de Versailles, 45 avenue des États-Unis, 78035 Versailles Cedex, France; e-mail: canuto@math.uvsq.fr 
(i)

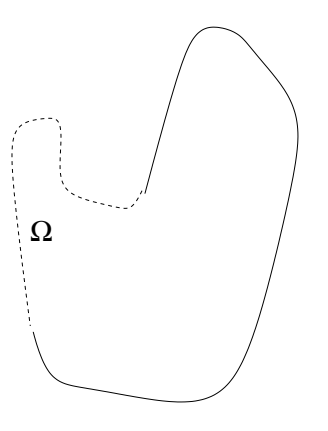

(ii)

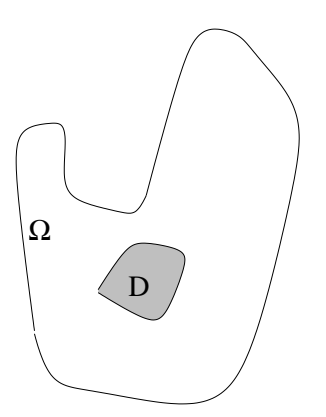

(iii)

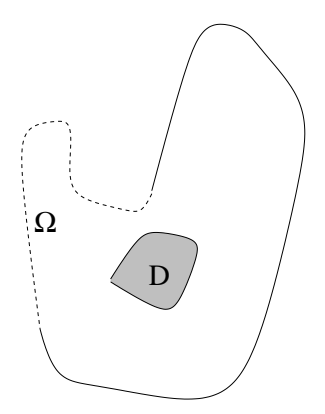

.... the inaccessible part of the boundary

- the accessible part of the boundary

$\checkmark$ the cavity

Figure 1. The pictures (i), (ii), (iii) show the three different situations.

thermal diffusion coefficient, that is $\kappa$ is a symmetric $N \times N$ matrix-valued function in $\bar{\Omega}$ satisfying the following conditions:

(i) there exists a constant $\alpha>0$, such that for all $x \in \bar{\Omega}$, and for all $\xi \in \mathbb{R}^{N}$,

$$
\kappa(x) \xi \cdot \xi \geq \alpha|\xi|^{2} \quad(\text { ellipticity })
$$

(ii) there exists a constant $C \geq 0$, such that for all $x, y \in \bar{\Omega}$,

$$
|\kappa(x)-\kappa(y)| \leq C|x-y| \quad(\text { Lipschitz continuity) }
$$

For $\Omega, D, \kappa, u_{0}, \varphi, \psi, g$ assigned, suppose that $u$ solves the following parabolic problem, which we call the direct problem:

$$
\left\{\begin{array}{rlll}
\partial_{t} u-\operatorname{div}(\kappa(x) \nabla u) & =0 & & \text { in } \quad(0, T) \times \Omega \backslash \bar{D} \\
u(0) & =u_{0} & & \text { in } \quad \Omega \backslash \bar{D} \\
\kappa \nabla u \cdot \mathbf{n} & =\varphi(t, \sigma) & & \text { on } \quad(0, T) \times \partial D \\
\kappa \nabla u \cdot \mathbf{n} & =\psi(t, \sigma) & & \text { on } \quad(0, T) \times I, \\
\kappa \nabla u \cdot \mathbf{n} & =g(t, \sigma) & & \text { on } \quad(0, T) \times A
\end{array}\right.
$$

where $\mathbf{n}$ denotes the outer unit normal at $\partial(\Omega \backslash D)$. Here and in the sequel $I$ is a relatively open piece of $\partial \Omega$. It is well-known that, under reasonable assumptions on the data, problem (1.3) has a unique solution, and that the temperature $u(t, \sigma)$ is well-defined for $(t, \sigma) \in(0, T) \times \partial \Omega$. In the present paper we are interested in the following problem:

Let $\Lambda$ be the so-called input-output map, that is

$$
\Lambda: g \longmapsto u\left(T_{0}\right)_{\mid A_{\text {out }}},
$$


where $T_{0} \in(0, T]$ is a given fixed time and $A_{\text {out }}$ is a relatively open piece of $A$, and let $\Phi$ be the operator

$$
\Phi:(I, D) \longmapsto \Lambda \text {. }
$$

Is $\Phi$ injective?

We point out that to prove the injectivity of the operator $\Phi$ is equivalent to show the uniqueness of $I$ and $D$ from knowledge of all possible pairs of input-output data

$$
\left(g, u\left(T_{0}\right)_{\mid A_{\text {out }}}\right)
$$

of the solution $u$ of (1.3), that is from all possible measurements of temperature $u\left(T_{0}\right)_{\mid A_{\text {out }}}$ at a given time $T_{0}$ on $A_{\text {out }}$. We observe moreover that the temperature is measured at a given time $T_{0}$ only, instead of measuring it over a whole interval of time such as $\left[0, T_{0}\right]$. We study also the problem in which a mean value of the temperature is measured over a small interval of time. We note finally that the initial temperature $u_{0}$, and the boundary data $\varphi, \psi$ in (1.3) are given arbitrarily. This assumption corresponds to a real situation in which the data $u_{0}$, $\varphi, \psi$ are a priori unknown.

A similar problem has been investigated by Vessella [8]. He proves the unique localization of a thermic insulating region $D$ in $\Omega$ (in (1.3) he supposes $\kappa \nabla u \cdot \mathbf{n}=0$ on $(0, T) \times \partial D, I$ known, and $A=\partial \Omega$ ) from a single measurement of temperature $u_{\mid\left(t_{0}, t_{1}\right) \times A_{\text {out }}}$ on $\left[t_{0}, t_{1}\right] \times A_{\text {out }}$, where $\left(t_{0}, t_{1}\right)$ is a subinterval of $[0, T]$, and $A_{\text {out }}$ is a relatively open piece of $\partial \Omega$, provided that $\Omega$ is a contractible domain, the initial temperature $u_{0}$ in $(1.3)$ is constant, and the input $g$ is monotone with respect to the time variable $t$. Vessella's proof is based on the unique continuation principle and the maximum principle for parabolic equations. Moreover Vessella shows, when $N=3$, and $\kappa=I_{3}$ ( $I_{3}$ is the $3 \times 3$ identity matrix), a continuous dependence of logarithmic type of the domain $D$ from the temperature $u_{\mid\left(t_{0}, t_{1}\right) \times A_{\text {out }}}$.

In order to prove the injectivity of the operator $\Phi$ in (1.5), we will inspire with the so-called boundary spectral data method, introduced in [2] by the author and Kavian to show the identifiability of coefficients in a class of heat equations via boundary measurements. This method consists in studying the identifiability of the boundary spectral data for the underlying elliptic operator in (1.3) from the input-output map $\Lambda$. More precisely let $\left(\lambda_{k}\right)_{k=1}^{\infty},\left(\varphi_{k}\right)_{k=1}^{\infty}$ be respectively the nondecreasing sequence of eigenvalues and the corresponding eigenfunctions of the problem (with Neumann boundary conditions):

$$
\left\{\begin{aligned}
-\operatorname{div}\left(\kappa \nabla \varphi_{k}\right) & =\lambda_{k} \varphi_{k} & & \text { in } \Omega \backslash \bar{D}, \\
\kappa \nabla \varphi_{k} \cdot \mathbf{n} & =0 & & \text { on } \partial D, \\
\kappa \nabla \varphi_{k} \cdot \mathbf{n} & = & & \text { on } \partial \Omega, \\
\int_{\Omega \backslash D}\left|\varphi_{k}\right|^{2} \mathrm{~d} x & = & &
\end{aligned}\right.
$$

Let us denote by $\operatorname{DBSD}(I, D)$ the so-called Dirichlet Boundary Spectral Data, i.e.

$$
\operatorname{DBSD}(I, D):=\left(\lambda_{k}, \varphi_{k \mid A}\right)_{k=1}^{\infty} .
$$

The question we ask is the following:

Does the input-output map $\Lambda$ determine the Dirichlet Boundary Spectral Data $\operatorname{DBSD}(I, D)$ uniquely?

The first result of the present paper is the following:

Theorem 1.1. For an integer $N \geq 2$, let $\Omega_{j}$, for $j \in\{0,1\}$, be two bounded domains in $\mathbb{R}^{N}$ of class $C^{0,1}$, having common boundary $A:=\left(\partial \Omega_{j}\right) \backslash I_{j}, I_{j}$ being a relatively open piece of $\partial \Omega_{j}$, and let $D_{j}$ be a $C^{0,1}$ domains such 


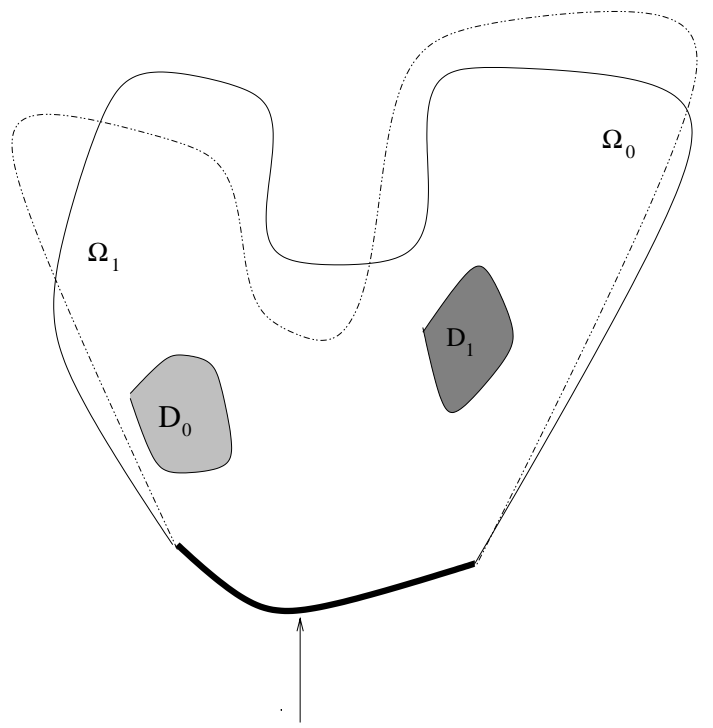

A: the part accessible to observations

Figure 2. The domains $\Omega_{0}, \Omega_{1}$, and the cavities $D_{0}, D_{1}$.

that the closure $\overline{D_{j}} \subset \Omega_{j}$. Let us denote by $\kappa$ a symmetric $N \times N$ matrix-valued function in $\overline{\Omega_{0}} \cup \overline{\Omega_{1}}$ satisfying conditions (1.1, 1.2) in $\overline{\Omega_{0}} \cup \overline{\Omega_{1}}$. Let $u_{j 0} \in H^{1}\left(\Omega_{j} \backslash D_{j}\right), \varphi_{j} \in C\left([0, T] ; L^{2}\left(\partial D_{j}\right)\right), \psi_{j} \in C\left([0, T] ; L^{2}\left(I_{j}\right)\right)$, $g \in C\left([0, T] ; L^{2}(A)\right)$ be such that $u_{j} \in C\left((0, T] ; H^{1}\left(\Omega_{j} \backslash D_{j}\right)\right) \cap C^{1}\left([0, T] ; L^{2}\left(\Omega_{j} \backslash D_{j}\right)\right)$ solve (1.3), when $\Omega:=\Omega_{j}$, and $D:=D_{j}$. Suppose that

$$
\Lambda_{0}(g)=\Lambda_{1}(g) \text { in } L^{2}\left(A_{\text {out }}\right),
$$

where $\Lambda_{j}(g):=u_{j}\left(T_{0}\right)_{\mid A_{\text {out }}}$, for all $g \in C\left([0, T] ; L^{2}(A)\right)$ such that the $\operatorname{supp}(g(t, \cdot)) \subset A$ for $t \in[0, T]$. Then the Dirichlet Boundary Spectral Data $\operatorname{DBSD}\left(I_{j}, D_{j}\right)(1.7)$ (when $\Omega:=\Omega_{j}$, and $D:=D_{j}$ in (1.6)) coincide, that is, up to an appropriate choice of the eigenfunctions $\varphi_{0 k}$, for all $k \geq 1$, one has

$$
\lambda_{0 k}=\lambda_{1 k}, \quad \text { and } \quad \varphi_{0 k}=\varphi_{1 k} \text { a.e on } A .
$$

We point out that the conclusion of Theorem 1.1 remains valid if we replace hypothesis (1.8) by equality of the mean values of the temperatures in the interval $\left[\tau_{0}-T_{0}, T_{0}\right]$, that is the following result holds:

Theorem 1.2. Let $0<\tau_{0}<T_{0}$ be given. Under the assumptions of Theorem 1.1 assume that

$$
\int_{T_{0}-\tau_{0}}^{T_{0}} u_{0}(t)_{\mid A_{\text {out }}} \mathrm{d} t=\int_{T_{0}-\tau_{0}}^{T_{0}} u_{1}(t)_{\mid A_{\text {out }}} \mathrm{d} t \quad \text { in } L^{2}\left(A_{\text {out }}\right),
$$

for all $g \in C\left([0, T] ; L^{2}(A)\right)$ such that the $\operatorname{supp}(g(t, \cdot)) \subset A$ for $t \in[0, T]$. Then the Dirichlet Boundary Spectral Data $\operatorname{DBSD}\left(I_{j}, D_{j}\right)(1.7)$ coincide, that is, up to an appropriate choice of the eigenfunctions $\varphi_{0 k}$, for all $k \geq 1$, one has that

$$
\lambda_{0 k}=\lambda_{1 k}, \quad \text { and } \varphi_{0 k}=\varphi_{1 k} \quad \text { a.e. on } A \text {. }
$$


We now consider the Dirichlet case. More precisely let $\left(\mu_{k}\right)_{k=1}^{\infty},\left(\psi_{k}\right)_{k=1}^{\infty}$ be respectively the nondecreasing sequence of eigenvalues and the corresponding eigenfunctions of the following problem (with Dirichlet boundary conditions):

$$
\left\{\begin{aligned}
-\operatorname{div}\left(\kappa \nabla \psi_{k}\right) & =\mu_{k} \psi_{k} & & \text { in } \Omega \backslash \bar{D} \\
\psi_{k} & =0 & & \text { on } \partial D \\
\psi_{k} & =0 & & \text { on } \partial \Omega \\
\int_{\Omega \backslash D}\left|\psi_{k}\right|^{2} \mathrm{~d} x & = & &
\end{aligned}\right.
$$

Let us denote by $\operatorname{NBSD}(I, D)$ the so-called Neumann Boundary Spectral Data, i.e.

$$
\operatorname{NBSD}(I, D):=\left(\mu_{k}, \kappa \nabla \psi_{k} \cdot \mathbf{n}_{\mid A}\right)_{k=1}^{\infty} .
$$

Then the conclusion of Theorem 1.1 remains valid if in (1.3) we substitute the Neumann boundary conditions with the corresponding Dirichlet boundary conditions. More precisely the following result holds:

Theorem 1.3. Under the hypothesis of Theorem 1.1, assume that $A:=\left(\partial \Omega_{j}\right) \backslash I_{j}$ is of class $C^{1,1}$. Let $u_{j 0} \in H^{1}\left(\Omega_{j} \backslash D_{j}\right), \varphi_{j} \in C\left([0, T] ; H^{\frac{1}{2}}\left(\partial D_{j}\right)\right), \psi_{j} \in C\left([0, T] ; H^{\frac{1}{2}}\left(I_{j}\right)\right), f \in C\left([0, T] ; H^{\frac{3}{2}}(A)\right)$ be such that $u_{j} \in C\left((0, T] ; H^{1}\left(\Omega_{j} \backslash D_{j}\right)\right) \cap C^{1}\left([0, T] ; L^{2}\left(\Omega_{j} \backslash D_{j}\right)\right)$ solve

$$
\left\{\begin{aligned}
\partial_{t} u_{j}-\operatorname{div}\left(\kappa(x) \nabla u_{j}\right) & =0 & & \text { in }(0, T) \times \Omega_{j} \backslash \overline{D_{j}}, \\
u_{j}(0) & =u_{j 0} & & \text { in } \Omega_{j} \backslash \overline{D_{j}}, \\
u_{j} & =\varphi_{j}(t, \sigma) & & \text { on }(0, T) \times \partial D_{j}, \\
u_{j} & =\psi_{j}(t, \sigma) & & \text { on }(0, T) \times I_{j}, \\
u_{j} & =f(t, \sigma) & & \text { on }(0, T) \times A .
\end{aligned}\right.
$$

We denote by

$$
\widetilde{\Lambda}_{j}(f):=\kappa \nabla u_{j}\left(T_{0}\right) \cdot \mathbf{n}_{\mid A_{\text {out }}}
$$

the thermal fluxes measured at a given time $T_{0} \in(0, T]$ on $A_{\text {out }}$. Suppose that

$$
\widetilde{\Lambda}_{0}(f)=\widetilde{\Lambda}_{1}(f) \text { in } L^{2}\left(A_{\text {out }}\right)
$$

for all $f \in C\left([0, T] ; H^{\frac{3}{2}}(A)\right)$ such that the $\operatorname{supp}(f(t, \cdot) \subset$ A for $t \in[0, T]$. Then the Neumann Boundary Spectral Data $\operatorname{NBSD}\left(I_{j}, D_{j}\right)$ (1.11) (when $\Omega:=\Omega_{j}$, and $D:=D_{j}$ in (1.10)) coincide, that is, up to an appropriate choice of the eigenfunctions $\psi_{0 k}$, for all $k \geq 1$, one has:

$$
\mu_{0 k}=\mu_{1 k}, \quad \text { and } \quad \kappa \nabla \psi_{0 k} \cdot \mathbf{n}=\kappa \nabla \psi_{1 k} \cdot \mathbf{n} \quad \text { a.e on } A .
$$

Remark 1.4. Theorem 1.3 holds true if we replace hypothesis (1.14) by equality of the mean values of the fluxes in the interval $\left[\tau_{0}-T_{0}, T_{0}\right]$, i.e. we suppose that, for a fixed $\tau_{0}, 0<\tau_{0}<T_{0}$,

$$
\int_{T_{0}-\tau_{0}}^{T_{0}} \kappa \nabla u_{0}(t) \cdot \mathbf{n}_{\mid A_{\text {out }}} \mathrm{d} t=\int_{T_{0}-\tau_{0}}^{T_{0}} \kappa \nabla u_{1}(t) \cdot \mathbf{n}_{\mid A_{\text {out }}} \mathrm{d} t \quad \text { in } L^{2}\left(A_{\text {out }}\right),
$$

for all $f \in C\left([0, T] ; H^{\frac{3}{2}}(A)\right)$ such that the support $\operatorname{supp}(f(t, \cdot)) \subset A$ for $t \in[0, T]$. 
Once the result of Theorem 1.1 is at hand, we can prove the injectivity of the operator $\Phi$ defined in (1.5). This is proved in the following:

Theorem 1.5. Under the assumptions of Theorem 1.1, for $N=2$, 3, suppose that the Dirichlet Boundary Spectral Data $\operatorname{DBSD}\left(I_{j}, D_{j}\right)$ (1.7) (when $\Omega:=\Omega_{j}$, and $D:=D_{j}$ in (1.6)) coincide, that is, for all $k \geq 1$,

$$
\lambda_{0 k}=\lambda_{1 k}, \text { and } \varphi_{0 k}=\varphi_{1 k} \quad \text { a.e. on } A .
$$

Then $I_{0}=I_{1}$ and $D_{0}=D_{1}$.

The conclusion of Theorem 1.5 remains valid if we assume that the Neumann Boundary Spectral Data $\operatorname{NBSD}\left(I_{j}, D_{j}\right)$ coincide:

Theorem 1.6. Under the assumptions of Theorem 1.3, for $N=2,3$, suppose that the Neumann Boundary Spectral Data NBSD $\left(I_{j}, D_{j}\right)$ (1.11) (when $\Omega:=\Omega_{j}$, and $D:=D_{j}$ in (1.10)) coincide, that is, for all $k \geq 1$,

$$
\mu_{0 k}=\mu_{1 k}, \quad \kappa \nabla \psi_{0 k} \cdot \mathbf{n}=\kappa \nabla \psi_{1 k} \cdot \mathbf{n} \quad \text { a.e. on } A .
$$

Then $I_{0}=I_{1}$ and $D_{0}=D_{1}$.

The remainder of the paper is organized as follows: in Section 2 we gather some preliminary results and the notations used throughout; in Section 3 we prove Theorems 1.1-1.3; in Section 4 we prove Theorems 1.5 and 1.6 .

\section{Preliminary Results}

We denote by $\widetilde{\Omega}$ a bounded domain in $\mathbb{R}^{N}, N \geq 2$, with boundary of class $C^{0,1}$.

By $\kappa(x)$ we mean a symmetric $N \times N$ matrix-valued function in $\widetilde{\Omega}$ satisfying conditions $(1.1,1.2)$ in $\widetilde{\Omega}$.

We denote by $(L, \mathcal{D}(L))$ the elliptic operator

$$
L v:=-\operatorname{div}(\kappa(x) \nabla v)
$$

with domain

$$
\mathcal{D}(L):=\left\{v \in L^{2}(\widetilde{\Omega}) ; \quad L v \in L^{2}(\widetilde{\Omega}), \kappa \nabla v \cdot \mathbf{n}_{\mid \partial \widetilde{\Omega}}=0\right\} .
$$

Actually when $\kappa \in C^{0,1}(\overline{\widetilde{\Omega}})$ and $\partial \widetilde{\Omega} \in C^{0,1}$, then

$$
\mathcal{D}(L)=\left\{v \in H_{\mathrm{loc}}^{2}(\widetilde{\Omega}) \cap H^{1}(\widetilde{\Omega}) ; \kappa \nabla v \cdot \mathbf{n}_{\mid \partial \widetilde{\Omega}}=0\right\} .
$$

The operator $L$ possesses a sequence of eigenvalues $\left(\lambda_{k}\right)_{k=1}^{\infty}$ (which we suppose in a nondecreasing order) and corresponding eigenfunctions $\left(\varphi_{k}\right)_{k=1}^{\infty}$ satisfying:

$$
\left\{\begin{array}{rlrl}
-\operatorname{div}\left(\kappa \nabla \varphi_{k}\right) & =\lambda_{k} \varphi_{k} & & \text { in } \widetilde{\Omega}, \\
\kappa \nabla \varphi_{k} \cdot \mathbf{n} & =0 & & \text { on } \partial \widetilde{\Omega} \\
\int_{\widetilde{\Omega}}\left|\varphi_{k}\right|^{2} \mathrm{~d} x & =1, &
\end{array}\right.
$$


which form a Hilbert basis of $L^{2}(\widetilde{\Omega})$. We recall that the first eigenvalue $\lambda_{1}=0$, and the corresponding eigenfunction $\varphi_{1}(x)=|\widetilde{\Omega}|^{-\frac{1}{2}}$, where $|\cdot|$ denotes the Lebesgue measure of $\widetilde{\Omega}$. It is also known that the domain $\mathcal{D}(L)$ can be characterized by

$$
\mathcal{D}(L)=\left\{v \in L^{2}(\widetilde{\Omega}) ; \sum_{k=1}^{\infty} \lambda_{k}^{2}\left|\left(v \mid \varphi_{k}\right)\right|^{2}<+\infty\right\},
$$

where $(\cdot \mid \cdot)$ is the inner product in $L^{2}(\widetilde{\Omega})$.

We denote by $m_{k}$ the geometric multiplicity of $\lambda_{k}$. We recall that the eigenvalues $\lambda_{k}$ behave like

$$
\lambda_{k} \sim C_{0} k^{\frac{2}{N}} \quad \text { as } k \rightarrow+\infty,
$$

where the constant $C_{0}$ depends on $\kappa,|\widetilde{\Omega}|, N$ (see Courant and Hilbert [5], pp. 442-443). Moreover there exist two positive constants $C_{1}, C_{2}$ such that, for all $k \geq 1$, one has:

$$
C_{1} \lambda_{k} \leq\left\|\varphi_{k}\right\|_{H^{1}(\widetilde{\Omega})}^{2} \leq C_{2} \lambda_{k} .
$$

The following three lemmas, which are identical to Lemmas 2.1-2.3 respectively in Canuto and Kavian [2], are a tools to prove Theorem 1.1 later on. First we shall need the following result concerning the linear independence, or linear dependence, of the family $\left(\varphi_{k \mid \partial \tilde{\Omega}}\right)_{k \geq 1}$. In general these functions are not linearly independent. However one can show that the traces on the boundary of $\widetilde{\Omega}$ of eigenfunctions corresponding to a given eigenvalue $\lambda_{k_{0}}$ are actually independent. More precisely if $\lambda_{k}$ is an eigenvalue of $L$ having multiplicity $m_{k} \geq 1$, let us denote by $\varphi_{k, i}$ for $1 \leq i \leq m_{k}$ the eigenfunctions corresponding to the eigenvalue $\lambda_{k}$ which form a Hilbert basis of the kernel $N\left(L-\lambda_{k} I\right)$. We may state the following:

Lemma 2.1. For an integer $N \geq 2$, let $\widetilde{\Omega}$ be a bounded domain in $\mathbb{R}^{N}$ of class $C^{0,1}$. If, for a fixed $k \geq 1, \lambda_{k}$ is an eigenvalue of multiplicity $m_{k} \geq 1$ of $L$, and if $\Gamma$ is a relatively open piece of $\partial \widetilde{\Omega}$, then the dimension of the subspace spanned in $L^{2}(\Gamma)$ by $\left(\varphi_{k, i \mid \partial \tilde{\Omega}}\right)_{1 \leq i \leq m_{k}}$ is exactly $m_{k}$.

Proof of Lemma 2.1. Indeed if there exists $\left(c_{i}\right)_{i=1}^{m_{k}} \in \mathbb{R}^{m_{k}}$ such that

$$
\sum_{i=1}^{m_{k}} c_{i} \varphi_{k, i}=0 \quad \text { on } \Gamma
$$

then, setting $\varphi:=\sum_{i=1}^{m_{k}} c_{i} \varphi_{k, i}$, one checks that

$$
L \varphi=\lambda_{k} \varphi \quad \text { in } \widetilde{\Omega}, \quad \kappa \nabla \varphi \cdot \mathbf{n}=0 \quad \text { on } \partial \widetilde{\Omega}, \quad \varphi=0 \quad \text { on } \Gamma .
$$

Now, following a standard argument, we extend $\varphi \equiv 0$ in an exterior neighborhood of $\Gamma^{\prime} \subset \Gamma$. The unique continuation principle (see Garofalo and Lin [4]) implies then that $\varphi \equiv 0$ in $\widetilde{\Omega}$. Due to the fact that the functions $\varphi_{k, i}$ are linearly independent, we conclude that $c_{i}=0$ for $1 \leq i \leq m_{k}$. The proof of Lemma 2.1 is complete.

From this we conclude the following:

Lemma 2.2. Under the assumptions of Lemma 2.1, let $\Gamma_{1}$ and $\Gamma_{2}$ be two relatively open pieces of $\partial \widetilde{\Omega}$. For a fixed $k \geq 1$ consider the function $\Xi_{k}$ defined by

$$
\Xi_{k}\left(\sigma^{\prime}, \sigma\right):=\sum_{i=1}^{m_{k}} \varphi_{k, i}\left(\sigma^{\prime}\right) \varphi_{k, i}(\sigma) \quad \text { on } \Gamma_{1} \times \Gamma_{2} .
$$


Then $\Xi_{k}\left(\sigma^{\prime}, \sigma\right)$ is not identically zero on any relatively open subset of $\Gamma_{1} \times \Gamma_{2}$.

Proof of Lemma 2.2. By contradiction, let $\Gamma_{1}^{\prime}$ be a relatively open piece of $\Gamma_{1}$, and let $\Gamma_{2}^{\prime}$ be a relatively open piece of $\Gamma_{2}$ such that

$$
\Xi_{k}\left(\sigma^{\prime}, \sigma\right) \equiv 0 \quad \text { on } \Gamma_{1}^{\prime} \times \Gamma_{2}^{\prime}
$$

By Lemma 2.1, we have that (2.6) implies that $\varphi_{k, i} \equiv 0$ on $\Gamma_{1}^{\prime}$ for $i=1, \cdots, m_{k}$, and so by the unique continuation principle it follows that $\varphi_{k, i} \equiv 0$ on $\widetilde{\Omega}$, which leads to a contradiction.

We shall also need the following algebraic lemma:

Lemma 2.3. For two arbitrary integers $m, n \geq 1$, let $Z$ be a non empty set, and let $X, Y$ be two subsets of $Z$. Assume that $f_{i}: X \cup Y \rightarrow \mathbb{R}$ (for $1 \leq i \leq m$ ) and $g_{\ell}: X \cup Y \rightarrow \mathbb{R}$ (for $1 \leq \ell \leq n$ ) are functions such that

(i)

$$
\sum_{i=1}^{m} f_{i}(x) f_{i}(y)=\sum_{\ell=1}^{n} g_{\ell}(x) g_{\ell}(y) \quad \text { for }(x, y) \in X \times Y
$$

(ii) $X \cap Y$ contains infinitely many points;

(iii) $f_{i}$, for $1 \leq i \leq m$, (resp. $g_{\ell}$, for $1 \leq \ell \leq n$,) are not identically zero in $X \cap Y$;

(iv) $\left\{f_{1}, \ldots, f_{m}\right\}$ (resp. $\left.\left\{g_{1}, \ldots, g_{n}\right\}\right)$ are linearly independent in $X \cap Y$.

Then $m=n$, and denoting

$$
F(x):=\left(\begin{array}{c}
f_{1}(x) \\
\vdots \\
f_{m}(x)
\end{array}\right) \quad \text { and } \quad G(x):=\left(\begin{array}{c}
g_{1}(x) \\
\vdots \\
g_{n}(x)
\end{array}\right)
$$

there exists an $m \times m$ orthogonal matrix $M$ such that for all $z \in X \cup Y$ one has $F(z)=M G(z)$.

(Recall that by an orthogonal matrix $M$ we mean $M M^{*}=M^{*} M=I_{m}$, where $I_{m}$ is the $m \times m$ identity matrix.) For the reader's convenience we give the proof of Lemma 2.3, although is identical to which of Lemma 2.3 in [2].

Proof of Lemma 2.3. Let us denote by $V_{0}$ (resp. $V_{1}$ ) the space spanned by $\left\{f_{1}, \cdots, f_{m}\right\}$ (resp. $\left\{g_{1}, \cdots, g_{n}\right\}$ ). Since $f_{1}$ is not identically zero in $X \cap Y$, there exists $x_{1} \in X \cap Y$ such that $f_{1}\left(x_{1}\right) \neq 0$; then, $f_{2}$ being linearly independent of $f_{1}$, and $X \cap Y$ containing infinitely many points, there exists $x_{2} \in X \cap Y$ such that:

$$
\operatorname{det}\left(\begin{array}{ll}
f_{1}\left(x_{1}\right) & f_{2}\left(x_{1}\right) \\
f_{1}\left(x_{2}\right) & f_{2}\left(x_{2}\right)
\end{array}\right) \neq 0
$$

By induction one sees that we may find points $x_{1}, x_{2}, \ldots, x_{m}$ in $X \cap Y$ such that the $m \times m$ matrix

$$
P:=\left(\begin{array}{cccc}
f_{1}\left(x_{1}\right) & f_{2}\left(x_{1}\right) & \cdots & f_{m}\left(x_{1}\right) \\
f_{1}\left(x_{2}\right) & f_{2}\left(x_{2}\right) & \cdots & f_{m}\left(x_{2}\right) \\
\vdots & \vdots & \vdots & \vdots \\
f_{1}\left(x_{m}\right) & f_{2}\left(x_{m}\right) & \cdots & f_{m}\left(x_{m}\right)
\end{array}\right)
$$


is invertible. So, setting $x=x_{j}$ in $(2.7)$, it follows that $P F(y)=\widetilde{P} G(y)$ in $Y$, where $\widetilde{P}$ is the following $m \times n$ matrix

$$
\widetilde{P}:=\left(\begin{array}{cccc}
g_{1}\left(x_{1}\right) & g_{2}\left(x_{1}\right) & \cdots & g_{n}\left(x_{1}\right) \\
g_{1}\left(x_{2}\right) & g_{2}\left(x_{2}\right) & \cdots & g_{n}\left(x_{2}\right) \\
\vdots & \vdots & \vdots & \vdots \\
g_{1}\left(x_{m}\right) & g_{2}\left(x_{m}\right) & \cdots & g_{n}\left(x_{m}\right)
\end{array}\right)
$$

From this it follows that $F(y)=P^{-1} \widetilde{P} G(y)$ for all $y \in Y$, where $P^{-1}$ is the inverse matrix of $P$.

Similarly, changing the role of the variables $x$ and $y$, we obtain that $F(x)=P^{-1} \widetilde{P} G(x)$ in $X$, that is

$$
F(z)=M G(z) \quad \text { in } X \cup Y
$$

where $M:=P^{-1} \widetilde{P}$. Therefore, recalling that the functions $\left\{f_{1}, \ldots, f_{m}\right\}$ and $\left\{g_{1}, \ldots, g_{n}\right\}$ are linearly independent in $X \cap Y$, it follows that $V_{0} \subseteq V_{1}$, that is $m \leq n$. In the same way one may prove that $n \leq m$, and so we conclude that $m=n$.

Finally we prove that the matrix $M=P^{-1} \widetilde{P}$ is orthogonal. Indeed, recalling that $F(z)=M G(z)$ for all $z \in X \cup Y$ and using (2.7), we obtain

$$
\left(M^{*} M-I_{m}\right) G(x) \cdot G(y)=0 \quad \text { in } X \times Y
$$

where $a \cdot b$ denotes the euclidean scalar product in $\mathbb{R}^{m}, M^{*}$ is the transpose matrix of $M$, and $I_{m}$ is the $m \times m$ identity matrix. Since the functions $\left\{g_{1}, \cdots, g_{m}\right\}$ are linearly independent in $X \cap Y$ it follows that $M^{*} M=M M^{*}=I_{m}$, that is $M$ is orthogonal.

The proof of Lemma 2.3 is complete.

\section{Proof of Theorems $1.1,1.2$ And 1.3}

The first task in this section is to prove Theorem 1.1. Before doing so we need to establish some preliminary lemmas.

Lemma 3.1. For an integer $N \geq 2$, let $\widetilde{\Omega}$ be a bounded domain in $\mathbb{R}^{N}$ of class $C^{0,1}$. For $g \in C\left([0, T] ; L^{2}(\partial \widetilde{\Omega})\right)$, let $u \in C\left((0, T] ; H^{1}(\widetilde{\Omega})\right) \cap C^{1}\left([0, T] ; L^{2}(\widetilde{\Omega})\right)$ solve

$$
\left\{\begin{array}{rlll}
\partial_{t} u-\operatorname{div}(\kappa(x) \nabla u) & =0 & \text { in }(0, T) \times \widetilde{\Omega} \\
u(0) & =0 & \text { in } \widetilde{\Omega} \\
\kappa \nabla u \cdot \mathbf{n} & =g & \text { on }(0, T) \times \partial \widetilde{\Omega}
\end{array}\right.
$$

where $\kappa$ satisfies assumptions $(1.1,1.2)$ in $\widetilde{\Omega}$. Then $u$ can be written in the following Fourier expansion:

$$
u(t)=\sum_{k=1}^{\infty} \alpha_{k}(t) \varphi_{k} \quad \text { in } L^{2}(\widetilde{\Omega}),
$$

where $\varphi_{k}$ is defined in (2.3), and

$$
\alpha_{k}(t):=-\int_{0}^{t} \int_{\partial \widetilde{\Omega}} \varphi_{k}\left(\sigma^{\prime}\right) \mathrm{e}^{-\lambda_{k}(t-\tau)} g\left(\tau, \sigma^{\prime}\right) \mathrm{d} \sigma^{\prime} \mathrm{d} \tau .
$$


Proof of Lemma 3.1. Since $\left(\varphi_{k}\right)_{k=1}^{\infty}$ is a Hilbert basis in $L^{2}(\widetilde{\Omega})$, we can write $u$ in the following Fourier expansion:

$$
u(t)=\sum_{k=1}^{\infty} \alpha_{k}(t) \varphi_{k} \quad \text { in } L^{2}(\widetilde{\Omega}),
$$

where $\alpha_{k}(t):=\left(u(t) \mid \varphi_{k}\right)$. Multiplying the equation in (3.1) by $\varphi_{k}$, and integrating by parts over $\widetilde{\Omega}$, we obtain, for all $k \geq 1$,

$$
\left\{\begin{aligned}
\alpha_{k}^{\prime}(t)+\lambda_{k} \alpha_{k}(t) & =-\int_{\partial \widetilde{\Omega}} \varphi_{k}\left(\sigma^{\prime}\right) g\left(t, \sigma^{\prime}\right) \mathrm{d} \sigma^{\prime} \text { in }(0, T) \\
\alpha_{k}(0) & =0
\end{aligned}\right.
$$

where $\alpha_{k}^{\prime}(t)$ denotes the derivative of $\alpha_{k}(t)$. The thesis of the lemma follows then trivially.

Lemma 3.2. Under the assumptions of Lemma 3.1, let $g$ satisfy the following condition:

$$
g \equiv 0 \quad \text { on }\left[T_{0}-\varepsilon_{0}, T_{0}\right] \times \partial \widetilde{\Omega},
$$

where $T_{0} \in(0, T]$, and $0<\varepsilon_{0}<T_{0}$. Then $u\left(T_{0}\right)_{\mid \partial \widetilde{\Omega}}$ can be written in the following way

$$
u\left(T_{0}\right)_{\mid \partial \widetilde{\Omega}}=-\left(\int_{0}^{T_{0}-\varepsilon_{0}} \int_{\partial \widetilde{\Omega}} \sum_{k=1}^{\infty} \varphi_{k}\left(\sigma^{\prime}\right) \mathrm{e}^{-\lambda_{k}\left(T_{0}-\tau\right)} g\left(\tau, \sigma^{\prime}\right) \mathrm{d} \sigma^{\prime} \mathrm{d} \tau\right) \varphi_{k \mid \partial \widetilde{\Omega}} \quad \text { in } H^{\frac{1}{2}}(\partial \widetilde{\Omega}) .
$$

Proof of Lemma 3.2. We divide the proof into two steps:

Step 1. If $g$ satisfies (3.5), then $u\left(T_{0}\right) \in \mathcal{D}(L)$, where $\mathcal{D}(L)$ is defined in (2.2). So it follows that (see (2.4))

$$
\sum_{k=1}^{\infty} \lambda_{k}^{2}\left|\alpha_{k}\left(T_{0}\right)\right|^{2}<+\infty
$$

where $\alpha_{k}\left(T_{0}\right):=\left(u\left(T_{0}\right) \mid \varphi_{k}\right)$. Setting $u_{m}\left(T_{0}\right):=\sum_{k=1}^{m} \alpha_{k}\left(T_{0}\right) \varphi_{k}$, then

$$
u_{m}\left(T_{0}\right) \rightarrow u\left(T_{0}\right) \quad \text { in } L^{2}(\widetilde{\Omega}) \quad \text { as } m \rightarrow+\infty
$$

and

$$
L u_{m}\left(T_{0}\right)-L u\left(T_{0}\right) \rightarrow 0 \quad \text { in } L^{2}(\widetilde{\Omega}) \quad \text { as } m \rightarrow+\infty
$$

By the fact that $\left\|u_{m}\left(T_{0}\right)\right\|_{H^{1}(\widetilde{\Omega})} \leq C\left\|L u_{m}\left(T_{0}\right)\right\|_{L^{2}(\widetilde{\Omega})}$, we have

$$
u\left(T_{0}\right)=\sum_{k=1}^{\infty} \alpha_{k}\left(T_{0}\right) \varphi_{k} \quad \text { in } H^{1}(\widetilde{\Omega}) .
$$


Now, since the trace operator $\gamma: u\left(T_{0}\right) \rightarrow u\left(T_{0}\right)_{\mid \partial \widetilde{\Omega}}$ is continuous from $H^{1}(\widetilde{\Omega})$ to $H^{\frac{1}{2}}(\partial \widetilde{\Omega})$, one has, in the sense of $H^{\frac{1}{2}}(\partial \widetilde{\Omega})$ :

$$
u\left(T_{0}\right)_{\mid \partial \widetilde{\Omega}}=-\sum_{k=1}^{\infty}\left(\int_{0}^{T_{0}-\varepsilon_{0}} \int_{\partial \widetilde{\Omega}} \varphi_{k}\left(\sigma^{\prime}\right) \mathrm{e}^{-\lambda_{k}\left(T_{0}-\tau\right)} g\left(\tau, \sigma^{\prime}\right) \mathrm{d} \sigma^{\prime} \mathrm{d} \tau\right) \varphi_{k \mid \partial \widetilde{\Omega}} .
$$

Step 2. In this step we prove that we can commute the series sign with the integral signs in the right hand side of (3.7). By Fubini's theorem it is sufficient, for example, to show that

$$
\mathcal{I}:=\int_{0}^{T_{0}-\varepsilon_{0}} \sum_{k=1}^{\infty}\left\|\varphi_{k}\right\|_{H^{\frac{1}{2}}(\partial \widetilde{\Omega})} \int_{\partial \widetilde{\Omega}}\left|\varphi_{k}\left(\sigma^{\prime}\right) \mathrm{e}^{-\lambda_{k}\left(T_{0}-\tau\right)} g\left(\tau, \sigma^{\prime}\right)\right| \mathrm{d} \sigma^{\prime} \mathrm{d} \tau<+\infty .
$$

In fact, denoting by $\langle\cdot, \cdot\rangle$ the duality $H^{-\frac{1}{2}}(\partial \widetilde{\Omega}), H^{\frac{1}{2}}(\partial \widetilde{\Omega})$, we have

$$
\begin{aligned}
\mathcal{I} & \leq \int_{0}^{T_{0}-\varepsilon_{0}} \sum_{k=1}^{\infty}\left|\left\langle\varphi_{k}, g(\tau)\right\rangle\right|\left\|\varphi_{k}\right\|_{H^{\frac{1}{2}}(\partial \widetilde{\Omega})} \mathrm{e}^{-\lambda_{k} \varepsilon_{0}} \mathrm{~d} \tau \\
& \leq \int_{0}^{T_{0}-\varepsilon_{0}}\|g(\tau)\|_{H^{-\frac{1}{2}}(\partial \widetilde{\Omega})} \mathrm{d} \tau \sum_{k=1}^{\infty}\left\|\varphi_{k}\right\|_{H^{\frac{1}{2}}(\partial \widetilde{\Omega})}^{2} \mathrm{e}^{-\lambda_{k} \varepsilon_{0}} \\
& \leq C \sum_{k=1}^{\infty} \lambda_{k} \mathrm{e}^{-\lambda_{k} \varepsilon_{0}}
\end{aligned}
$$

where the last inequality is obtained upon using the fact that, by the trace inequality, and (2.5), we have:

$$
\left\|\varphi_{k}\right\|_{H^{\frac{1}{2}(\partial \widetilde{\Omega})}} \leq C\left\|\varphi_{k}\right\|_{H^{1}(\widetilde{\Omega})} \leq C \lambda_{k}^{\frac{1}{2}}
$$

Note that $\sum_{k=1}^{\infty} \lambda_{k} \mathrm{e}^{-\lambda_{k} \varepsilon_{0}}<+\infty$ since $\lambda_{k} \sim k^{\frac{2}{N}}$, as $k \rightarrow+\infty$. Therefore we may write equation (3.7) as:

$$
u\left(T_{0}\right)_{\mid \partial \widetilde{\Omega}}=-\left(\int_{0}^{T_{0}-\varepsilon_{0}} \int_{\partial \widetilde{\Omega}} \sum_{k=1}^{\infty} \varphi_{k}\left(\sigma^{\prime}\right) \mathrm{e}^{-\lambda_{k}\left(T_{0}-\tau\right)} g\left(\tau, \sigma^{\prime}\right) \mathrm{d} \sigma^{\prime} \mathrm{d} \tau\right) \varphi_{k \mid \partial \widetilde{\Omega}} \quad \text { in } H^{\frac{1}{2}}(\partial \widetilde{\Omega}) .
$$

The proof of Lemma 3.2 is complete.

Lemma 3.3. Under the assumptions of Theorem 1.1, for $j \in\{0,1\}$, let $\overline{u_{j}}$ be solutions of (1.3) when $\Omega:=\Omega_{j}$, and $D:=D_{j}$, with initial data $\overline{u_{j 0}} \equiv 0$ in $\Omega_{j} \backslash \overline{D_{j}}$, and boundary data $\overline{\varphi_{j}} \equiv 0$ on $(0, T) \times \partial D_{j}$, and $\overline{\psi_{j}} \equiv 0$ on $(0, T) \times I_{j}$. Then

$$
\overline{\Lambda_{0}}(g)=\overline{\Lambda_{1}}(g) \text { in } L^{2}\left(A_{\text {out }}\right)
$$

where $\overline{\Lambda_{j}}(g):=\overline{u_{j}}\left(T_{0}\right)_{\mid A_{\text {out }}}$, for all $g \in\left([0, T] ; L^{2}(A)\right)$ such that the $\operatorname{supp}(g(t, \cdot)) \subset A$ for $t \in[0, T]$.

Proof of Lemma 3.3. Following Rakesh and Symes [6], we put $\overline{u_{j}}(t, x):=u_{j}(t, x)-v_{j}(t, x)$, where $v_{j}$ solve $(1.3)$ when $\Omega:=\Omega_{j}, D:=D_{j}$, with data $v_{j}(0)=u_{j 0}$ in $\Omega_{j} \backslash \overline{D_{j}}, v_{j}=\varphi_{j}$ on $(0, T) \times \partial D_{j}, v_{j}=\psi_{j}$ on $(0, T) \times I_{j}$, 
and $v_{j} \equiv 0$ on $(0, T) \times A$. Then $\overline{u_{j 0}} \equiv 0$ in $\Omega_{j} \backslash \overline{D_{j}}, \overline{\varphi_{j}} \equiv 0$ on $(0, T) \times \partial D_{j}$, and $\overline{\psi_{j}} \equiv 0$ on $(0, T) \times I_{j}$. So, if we denote by $\overline{\Lambda_{j}}(g):=\overline{u_{j}}\left(T_{0}\right)_{\mid A_{\text {out }}}$, it follows that

$$
\overline{\Lambda_{j}}(g)=\Lambda_{j}(g)-\Lambda_{j}(0) .
$$

Hence $\overline{\Lambda_{0}}(g)=\overline{\Lambda_{1}}(g)$ for all $g \in C\left([0, T] ; L^{2}(A)\right)$ such that the support $\operatorname{supp}(g(t, \cdot)) \subset A$ for $t \in[0, T]$.

The proof of Lemma 3.3 is complete.

Lemma 3.4. Under the assumptions of Theorem 1.1, for all $k \geq 1$, we have

$$
\lambda_{0 k}=\lambda_{1 k},
$$

and

$$
\sum_{i=1}^{m_{0 k}} \varphi_{0 k, i}\left(\sigma^{\prime}\right) \varphi_{0 k, i}(\sigma)=\sum_{\ell=1}^{m_{1 k}} \varphi_{1 k, \ell}\left(\sigma^{\prime}\right) \varphi_{1 k, \ell}(\sigma)
$$

a.e. on $A \times A_{\text {out }}$.

(As we have mentioned in Sect. $2, m_{j k}$ is the multiplicity of the eigenvalue $\lambda_{j k}$.)

Proof of Lemma 3.4. First of all, using Lemma 3.3, we can always reduce to the case where the initial data $u_{j 0} \equiv 0$ in $\Omega_{j} \backslash \overline{D_{j}}$, and the boundary data $\varphi_{j} \equiv 0$ on $(0, T) \times \partial D_{j}$, and $\psi_{j} \equiv 0$ on $(0, T) \times I_{j}$. We recall that by hypothesis we have

$$
u_{0}\left(T_{0}\right)_{\mid A_{\text {out }}}=u_{1}\left(T_{0}\right)_{\mid A_{\text {out }}} \text { in } L^{2}\left(A_{\text {out }}\right),
$$

for all $g \in C\left([0, T] ; L^{2}(A)\right)$ such that the support $\operatorname{supp}(g(t, \cdot)) \subset A$ for $t \in[0, T]$. By Lemma 3.2 we know that if $g \equiv 0$ on $\left[T_{0}-\varepsilon_{0}, T_{0}\right] \times A$, then $u_{j}\left(T_{0}\right)_{\mid A_{\text {out }}}$ on $A_{\text {out }}$ can be written in the following way (in the sense of $\left.H^{\frac{1}{2}}\left(A_{\text {out }}\right)\right)$ :

$$
u_{j}\left(T_{0}\right)_{\mid A_{\text {out }}}=-\int_{0}^{T_{0}-\varepsilon_{0}} \int_{A} \sum_{k=1}^{\infty} \Phi_{j k}\left(\sigma^{\prime}, T_{0}-\tau\right) g\left(\tau, \sigma^{\prime}\right) \mathrm{d} \sigma^{\prime} \mathrm{d} \tau,
$$

where

$$
\Phi_{j k}\left(\sigma^{\prime}, \tau\right):=\sum_{k=1}^{\infty} \varphi_{j k}\left(\sigma^{\prime}\right) \mathrm{e}^{-\lambda_{j k} \tau} \varphi_{j k \mid A_{\text {out }}} .
$$

Then, from (3.9), it follows that

$$
\int_{0}^{T_{0}-\varepsilon_{0}} \int_{A} \sum_{k=1}^{\infty}\left(\Phi_{0 k}\left(\sigma^{\prime}, T_{0}-\tau\right)-\Phi_{1 k}\left(\sigma^{\prime}, T_{0}-\tau\right)\right) g\left(\tau, \sigma^{\prime}\right) \mathrm{d} \sigma^{\prime} \mathrm{d} \tau=0 \quad \text { in } L^{2}\left(A_{\text {out }}\right) .
$$

In particular we may assume that $g\left(\tau, \sigma^{\prime}\right) \equiv 0$ for $\tau \neq\left[T^{\prime}-\varepsilon^{\prime}, T^{\prime}+\varepsilon^{\prime}\right]$, and $\sigma^{\prime} \in A$, where $T^{\prime}$ is a fixed time, $T^{\prime} \in\left(0, T_{0}-\varepsilon_{0}\right)$, and $0<\varepsilon^{\prime}<T^{\prime}$. Then (3.11) becomes

$$
\int_{T^{\prime}-\varepsilon^{\prime}}^{T^{\prime}+\varepsilon^{\prime}} \int_{A} \sum_{k=1}^{\infty}\left(\Phi_{0 k}\left(\sigma^{\prime}, T_{0}-\tau\right)-\Phi_{1 k}\left(\sigma^{\prime}, T_{0}-\tau\right)\right) g\left(\tau, \sigma^{\prime}\right) \mathrm{d} \sigma^{\prime} \mathrm{d} \tau=0
$$


for all such functions $g$. Hence we have

$$
\sum_{k=1}^{\infty} \Phi_{0 k}\left(\sigma^{\prime}, \tau\right)=\sum_{k=1}^{\infty} \Phi_{1 k}\left(\sigma^{\prime}, \tau\right) \quad \text { in } L^{2}\left(A_{\text {out }}\right)
$$

for all $\sigma^{\prime} \in A, \tau \in\left[T^{\prime}-\varepsilon^{\prime}, T^{\prime}+\varepsilon^{\prime}\right]$. By the unique continuation principle for analytic functions of the variable $\tau$, we obtain

$$
\sum_{k=1}^{\infty} \Xi_{0 k}\left(\sigma^{\prime}\right) \mathrm{e}^{-\lambda_{0 k} \tau}=\sum_{k=1}^{\infty} \Xi_{1 k}\left(\sigma^{\prime}\right) \mathrm{e}^{-\lambda_{1 k} \tau} \quad \text { in } L^{2}\left(A_{\text {out }}\right)
$$

for all $\sigma^{\prime} \in A, \tau \in(0, \infty)$, where $\Xi_{j k}\left(\sigma^{\prime}\right):=\sum_{i=1}^{m_{j k}} \varphi_{j k, i}\left(\sigma^{\prime}\right) \varphi_{j k, i \mid A_{\text {out }}}, j \in\{0,1\}$. By Lemma 2.2 we know that, for all fixed $k \geq 1, \Xi_{j k}\left(\sigma^{\prime}\right)$ is not identically zero on any relatively open subset of $A$. Therefore, using the classical results on Dirichlet's series, equation (3.12) yields that, for all $k \geq 1$,

$$
\lambda_{0 k}=\lambda_{1 k}
$$

and

$$
\Xi_{0 k}=\Xi_{1 k} \text { in } L^{2}(A) \times L^{2}\left(A_{\text {out }}\right),
$$

that is

$$
\sum_{i=1}^{m_{0 k}} \varphi_{0 k, i}\left(\sigma^{\prime}\right) \varphi_{0 k, i}(\sigma)=\sum_{i=1}^{m_{1 k}} \varphi_{1 k, i}\left(\sigma^{\prime}\right) \varphi_{1 k, i}(\sigma)
$$

a.e. on $A \times A_{\text {out }}$. The proof of Lemma 3.4 is complete.

We are now in a position to prove Theorem 1.1.

Proof of Theorem 1.1. We prove that (3.13) implies that, for all $k \geq 1, m_{0 k}=m_{1 k}$, and, up to an appropriate choice of the eigenfunctions $\varphi_{0 k}, \varphi_{0 k}=\varphi_{1 k}$ a.e. on $A$.

For a fixed $k \geq 1$, let us note that, by Lemma $2.1, \varphi_{j k, i}$, for $i=1, \cdots, m_{j k}$, and $j \in\{0,1\}$, are linearly independent on $L^{2}(A)$. Now, applying the algebraic Lemma 2.3 with $m:=m_{0 k}, n:=m_{1 k}, Z=X:=A$, $Y:=A_{\text {out }}$,

$$
\begin{aligned}
& f_{i}:=\varphi_{0 k, i \mid A} \quad \text { for } 1 \leq i \leq m_{0 k}, \\
& g_{\ell}:=\varphi_{0 k, \ell \mid A} \quad \text { for } 1 \leq i \leq m_{1 k},
\end{aligned}
$$

and $F$ and $G$ respectively the vectors

$$
F:=\left(\begin{array}{c}
f_{1} \\
\vdots \\
f_{m_{0 k}}
\end{array}\right) \quad \text { and } \quad G:=\left(\begin{array}{c}
g_{1} \\
\vdots \\
g_{m_{1 k}}
\end{array}\right)
$$

we derive that $m_{0 k}=m_{1 k}$, and that there exists an $m \times m$ orthogonal matrix $M$, where $m:=m_{0 k}=m_{1 k}$, such that

$$
F(z)=M G(z) \text { for } z \in A
$$


B. CANUTO

We prove now that $\varphi_{0 k, i}=\varphi_{1 k, i}$ a.e. on $A$, for $i=1, \cdots, m$, up to an appropriate choice of the eigenfunctions $\varphi_{0 k, i}$. To prove this, let us define the vector

$$
\widetilde{\varphi_{0}}:=M^{*} \varphi_{0}^{*}
$$

where $M^{*}$ is the transpose matrix of $M$, that is $M_{i r}^{*}=M_{r i}$, and $\varphi_{0}^{*}$ is the transpose vector of $\varphi_{0}=$ $\left(\varphi_{0 k, 1}, \cdots, \varphi_{0 k, m}\right)$. First let us note that

$$
\left(\widetilde{\varphi_{0 k, i}} \mid \widetilde{\varphi_{0 k, \ell}}\right)=\delta_{i \ell} \text { for } 1 \leq i, \ell \leq m,
$$

where $(\cdot \mid \cdot)$ denotes the scalar product in $L^{2}\left(\Omega_{0} \backslash \overline{D_{0}}\right)$, and $\delta_{i \ell}$ is the Kronecker's symbol. In fact $\widetilde{\varphi_{0 k, i}}=$ $\sum_{r=1}^{m} M_{i r}^{*} \varphi_{0 k, r}$, and $\widetilde{\varphi_{0 k, \ell}}=\sum_{s=1}^{m} M_{\ell s}^{*} \varphi_{0 k, s}=\sum_{s=1}^{m} \varphi_{0 k, s} M_{s \ell}$, so

$$
\left(\widetilde{\varphi_{0 k, i}} \mid \widetilde{\varphi_{0 k, \ell}}\right)=\sum_{r=1}^{m} \sum_{s=1}^{m} M_{i r}^{*} M_{s \ell} \delta_{r s}=\sum_{r=1}^{m} M_{i r}^{*} M_{r \ell}=\delta_{i \ell}
$$

where the last equality follows since the matrix $M$ is orthogonal.

Now $\widetilde{\varphi_{0 k, i}}=\sum_{\ell=1}^{m} M_{i \ell}^{*} \varphi_{0 k, \ell}$, and, by (3.14), we know that $\varphi_{0 k, \ell}=\sum_{j=1}^{m} M_{\ell j} \varphi_{1 k, j}$ on $A$, so, substituting in $\widetilde{\varphi_{0 k, i}}$, we obtain

$$
\widetilde{\varphi_{0 k, i}}=\sum_{\ell=1}^{m} M_{i \ell}^{*} \sum_{j=1}^{m} M_{\ell j} \varphi_{1 k, j}=\sum_{j=1}^{m} \varphi_{1 k, j} \sum_{\ell=1}^{m} M_{i \ell}^{*} M_{\ell j}=\varphi_{1 k, i}
$$

on $A$, for $1 \leq i \leq m$, where the last equality follows since the matrix $M$ is orthogonal.

The proof of Theorem 1.1 is complete.

Now we prove Theorem 1.2.

Proof of Theorem 1.2. First of all, by Lemma 3.3, we can suppose that the initial data $u_{j 0} \equiv 0$ in $\Omega_{j} \backslash \overline{D_{j}}$, and the boundary data $\varphi_{j} \equiv 0$ on $(0, T) \times \partial D_{j}$, and $\psi_{j} \equiv 0$ on $(0, T) \times I_{j}$. Choosing $g$ such that $g \equiv 0$ on $\left[T_{0}-\varepsilon_{0}, T_{0}\right] \times A$, where $\varepsilon_{0}$ is such that $\tau_{0}<\varepsilon_{0}<T_{0}$, we can write $u_{j}$ in the following Fourier expansion (see $(3.2))$ :

$$
u_{j}(t)=-\sum_{k=1}^{\infty} \int_{0}^{T_{0}-\varepsilon_{0}} \int_{A} \varphi_{j k}\left(\sigma^{\prime}\right) \mathrm{e}^{-\lambda_{j k}(t-\tau)} g\left(\tau, \sigma^{\prime}\right) \mathrm{d} \sigma^{\prime} \mathrm{d} \tau \varphi_{j k} \quad \text { in } L^{2}\left(\Omega_{j} \backslash D_{j}\right),
$$

for $t \in\left[T_{0}-\tau_{0}, T_{0}\right]$. By Lemma 3.2 we obtain

$$
u_{j}(t)_{\mid A_{\text {out }}}=-\int_{0}^{T_{0}-\varepsilon_{0}} \int_{A} \sum_{k=1}^{\infty} \Phi_{j k}\left(\sigma^{\prime}, t-\tau\right) g\left(\tau, \sigma^{\prime}\right) \mathrm{d} \sigma^{\prime} \mathrm{d} \tau \quad \text { in } H^{\frac{1}{2}}\left(A_{\text {out }}\right)
$$

for $t \in\left[T_{0}-\tau_{0}, T_{0}\right]$, where $\Phi_{j k}$ are defined in (3.10). Now (1.9), and the change of variable $t-\tau=s$ in the right hand side of (3.15), imply

$$
\int_{T_{0}-\tau_{0}}^{T_{0}} \int_{t-T_{0}+\varepsilon_{0}}^{t} \int_{A} \sum_{k=1}^{\infty}\left(\Phi_{0 k}\left(\sigma^{\prime}, s\right)-\Phi_{1 k}\left(\sigma^{\prime}, s\right)\right) g\left(t-s, \sigma^{\prime}\right) \mathrm{d} \sigma^{\prime} \mathrm{d} s \mathrm{~d} t=0 .
$$


We may assume that the input $g\left(s, \sigma^{\prime}\right) \equiv 0$ for $s \notin\left[T^{\prime}-\varepsilon^{\prime}, T^{\prime}+\varepsilon^{\prime}\right]$, and $\sigma^{\prime} \in A$, where $T^{\prime} \in\left(\frac{T_{0}-\tau_{0}}{2}, \frac{T_{0}}{2}\right)$ is a fixed time, and $0<\varepsilon^{\prime}<T^{\prime}$. Then (3.16) becomes

$$
\int_{T_{0}-\tau_{0}}^{T_{0}} \int_{t-T^{\prime}-\varepsilon^{\prime}}^{t-T^{\prime}} \int_{A} \sum_{k=1}^{\infty}\left(\Phi_{0 k}\left(\sigma^{\prime}, s\right)-\Phi_{1 k}\left(\sigma^{\prime}, s\right)\right) g\left(t-s, \sigma^{\prime}\right) \mathrm{d} \sigma^{\prime} \mathrm{d} s \mathrm{~d} t=0
$$

for all such functions $g$. Hence it follows that

$$
\sum_{k=1}^{\infty} \Phi_{0 k}\left(\sigma^{\prime}, s\right)=\sum_{k=1}^{\infty} \Phi_{1 k}\left(\sigma^{\prime}, s\right) \quad \text { in } L^{2}\left(A_{\text {out }}\right)
$$

for all $\sigma^{\prime} \in A, s \in\left[T^{\prime}-\varepsilon^{\prime}, T^{\prime}+\varepsilon^{\prime}\right]$. By the unique continuation principle for analytic functions of the variable $s$ we obtain

$$
\sum_{k=1}^{\infty} \Xi_{0 k}\left(\sigma^{\prime}\right) \mathrm{e}^{-\lambda_{0 k} s}=\sum_{k=1}^{\infty} \Xi_{1 k}\left(\sigma^{\prime}\right) \mathrm{e}^{-\lambda_{1 k} s} \quad \text { in } L^{2}\left(A_{\text {out }}\right)
$$

for all $\sigma^{\prime} \in A, s \in(0, \infty)$, where $\Xi_{j k}\left(\sigma^{\prime}\right):=\sum_{i=1}^{m_{j k}} \varphi_{j k, i}\left(\sigma^{\prime}\right) \varphi_{j k, i \mid A_{\text {out }}}$. Repeating the same argument in order to prove Lemma 3.4 and Theorem 1.1, we obtain the thesis of Theorem 1.2.

Proof of Theorem 1.3. The proof is obtained similarly to the proof of Theorem 1.1, up to obvious changes (see also Canuto and Kavian [2]).

\section{Proof of TheOrems 1.5 AND 1.6}

In this section we prove Theorems 1.5 and 1.6.

Proof of Theorem 1.5. We divide the proof into two steps. In the first step we prove that $I_{0}=I_{1}$, in the second that $D_{0}=D_{1}$.

Step 1. By contradiction suppose that $I_{0} \neq I_{1}$. By hypothesis we know that the Dirichlet Boundary Spectral $\operatorname{Data} \operatorname{DBSD}\left(I_{j}, D_{j}\right), j \in\{0,1\}$, coincide, i.e.

$$
\lambda_{0 k}=\lambda_{1 k}=: \lambda_{k}, \quad \text { and } \quad \varphi_{0 k}=\varphi_{1 k}=: \varphi_{k} \quad \text { a.e. on } A .
$$

(We recall that $A:=\left(\partial \Omega_{j}\right) \backslash I_{j}$ is the common part of the boundaries of $\Omega_{j}$.) We denote by

$$
\omega_{0}:=\left(\Omega_{0} \backslash \overline{D_{0}}\right) \cap\left(\Omega_{1} \backslash \overline{D_{1}}\right),
$$

and $v_{k}:=\varphi_{0 k}-\varphi_{1 k}$ in $\omega_{0}$. Then, for all $k \geq 1, v_{k}$ solves

$$
\left\{\begin{aligned}
-\operatorname{div}\left(\kappa \nabla v_{k}\right) & =\lambda_{k} v_{k} & & \text { in } \omega_{0} \\
\kappa \nabla v_{k} \cdot \mathbf{n} & =0 & & \text { on } A, \\
v_{k} & =0 & & \text { on } A .
\end{aligned}\right.
$$

The unique continuation principle (see Garofalo and Lin [4]) implies that $v_{k} \equiv 0$ in $\overline{\omega_{0}}\left(\overline{\omega_{0}}\right.$ denotes the closure of $\left.\omega_{0}\right)$, that is

$$
\varphi_{0 k}=\varphi_{1 k} \quad \text { in } \overline{\omega_{0}},
$$




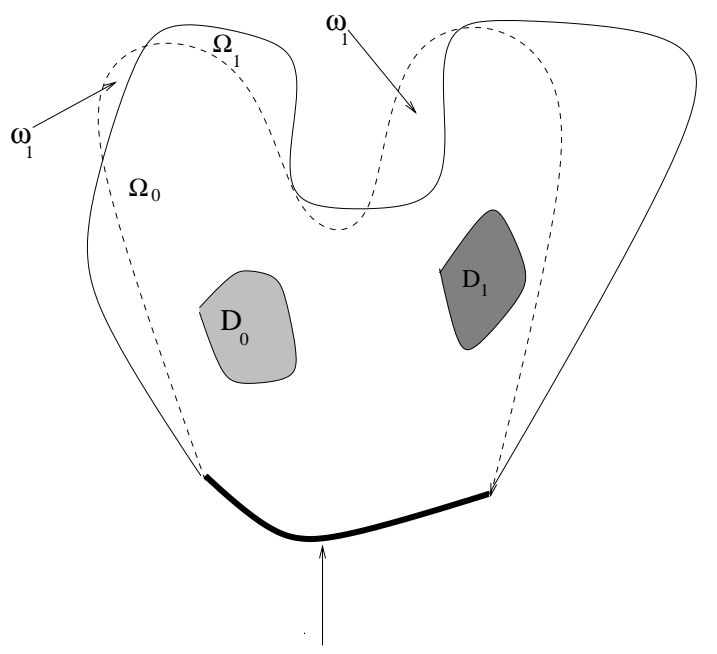

A: the part accessible to observations

Figure 3. The arrows show the domain $\omega_{1}$.

which yields that

$$
\kappa \nabla \varphi_{0 k} \cdot \mathbf{n}=\kappa \nabla \varphi_{1 k} \cdot \mathbf{n} \quad \text { on } \partial \omega_{0}
$$

Let us denote by

$$
\Omega:=\overline{\Omega_{0}} \cup \overline{\Omega_{1}}
$$

and

$$
\omega_{1}:=\Omega \backslash \overline{\Omega_{1}}
$$

Then Green's formula gives

$$
\int_{\omega_{1}} \varphi_{0 k} \mathrm{~d} x=-\frac{1}{\lambda_{k}} \int_{\partial \omega_{1}} \kappa \nabla \varphi_{0 k} \cdot \mathbf{n} \mathrm{d} \sigma
$$

for all $k \geq 2$. Since $\kappa \nabla \varphi_{j k} \cdot \mathbf{n}=0$ on $\partial \Omega_{j}$, for all $k \geq 1$, and using (4.1), it follows that $\int_{\partial \omega_{1}} \kappa \nabla \varphi_{0 k} \cdot \mathbf{n} \mathrm{d} \sigma=0$, i.e., for all $k \geq 2$,

$$
\int_{\omega_{1}} \varphi_{0 k} \mathrm{~d} x=0
$$

Let $\chi$ be the characteristic function of the set $\omega_{1}$ in $\Omega_{0} \backslash \overline{D_{0}}$, i.e.

$$
\chi(x)= \begin{cases}1 & \text { for } x \in \omega_{1} \\ 0 & \text { for } x \notin \omega_{1}\end{cases}
$$




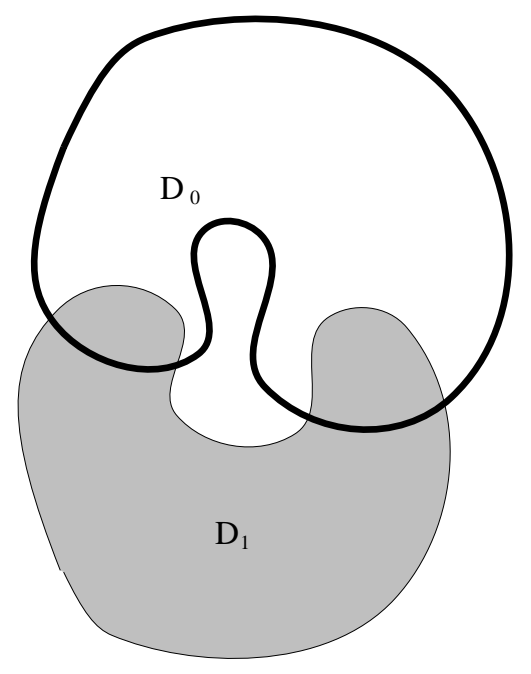

Figure 4. The picture shows the domains $D_{0}, D_{1}$.

By the fact that $\left(\varphi_{0 k}\right)_{k=1}^{\infty}$ is a Hilbert basis in $L^{2}\left(\Omega_{0} \backslash D_{0}\right)$, we have

$$
\chi=\sum_{k=1}^{\infty}\left(\chi \mid \varphi_{0 k}\right) \varphi_{0 k} \quad \text { in } L^{2}\left(\Omega_{0} \backslash D_{0}\right)
$$

where $\left(\chi \mid \varphi_{0 k}\right):=\int_{\Omega_{0} \backslash D_{0}} \chi \varphi_{0 k} \mathrm{~d} x=\int_{\omega_{1}} \varphi_{0 k} \mathrm{~d} x$. Since $\varphi_{01}(x):=\left|\Omega_{0} \backslash D_{0}\right|^{-\frac{1}{2}}$, and since by $(4.2) \int_{\omega_{1}} \varphi_{0 k} \mathrm{~d} x=0$ for all $k \geq 2$, it follows that $\chi=1$ in $\Omega_{0} \backslash \overline{D_{0}}$. This implies that $\omega_{1}=\Omega_{0} \backslash \overline{D_{0}}$, which leads to a contradiction. Then $I_{0}$ coincide with $I_{1}$.

Step 2. In this step we prove that $D_{0}=D_{1}$. First we prove that $D_{1} \subseteq D_{0}$. By contradiction suppose that $D_{1}$ is not contained in $D_{0}$. By the previous step $I_{0}=I_{1}$, so $\Omega_{0}=\Omega_{1}:=\Omega$. Let $D:=D_{0} \cup D_{1}$, and let $\omega$ be the connected component of $\Omega \backslash \bar{D}$ such that $\partial \Omega \subset \partial \omega$. Putting $v_{k}:=\varphi_{0 k}-\varphi_{1 k}$ in $\omega$, then $v_{k}$ solves:

$$
\left\{\begin{aligned}
-\operatorname{div}\left(\kappa \nabla v_{k}\right) & =\lambda_{k} v_{k} & & \text { in } \omega_{0} \\
\kappa \nabla v_{k} \cdot \mathbf{n} & =0 & & \text { on } \partial \Omega \\
v_{k} & =0 & & \text { on } A
\end{aligned}\right.
$$

By the unique continuation principle, it follows that $v_{k} \equiv 0$ in $\bar{\omega}(\bar{\omega}$ denotes the closure of $\omega)$, i.e.

$$
\varphi_{0 k}=\varphi_{1 k} \quad \text { in } \bar{\omega}
$$

which yields

$$
\kappa \nabla \varphi_{0 k} \cdot \mathbf{n}=\kappa \nabla \varphi_{1 k} \cdot \mathbf{n} \quad \text { on } \partial \omega
$$

Let us denote by

$$
\omega_{2}:=(\Omega \backslash \omega) \backslash \overline{D_{0}} .
$$


Green's formula gives

$$
\int_{\omega_{2}} \varphi_{0 k} \mathrm{~d} x=-\frac{1}{\lambda_{k}} \int_{\partial \omega_{2}} \kappa \nabla \varphi_{0 k} \cdot \mathbf{n} \mathrm{d} \sigma
$$

for all $k \geq 2$. By the fact that $\kappa \nabla \varphi_{j k} \cdot \mathbf{n}=0$ on $\partial\left(\Omega \backslash D_{j}\right)$, for all $k \geq 1$, and using (4.3), it follows that $\int_{\partial \omega_{2}} \kappa \nabla \varphi_{0 k} \cdot \mathbf{n} \mathrm{d} \sigma=0$, i.e., for all $k \geq 2$,

$$
\int_{\omega_{2}} \varphi_{0 k} \mathrm{~d} x=0
$$

Let $\chi$ be the characteristic function of the set $\omega_{2}$ in $\Omega \backslash \overline{D_{0}}$, i.e.

$$
\chi(x)= \begin{cases}1 & \text { for } x \in \omega_{2} \\ 0 & \text { for } x \notin \omega_{2} .\end{cases}
$$

Then

$$
\chi=\sum_{k=1}^{\infty}\left(\chi \mid \varphi_{0 k}\right) \varphi_{0 k} \quad \text { in } L^{2}\left(\Omega \backslash D_{0}\right),
$$

where $\left(\chi \mid \varphi_{0 k}\right):=\int_{\Omega \backslash D_{0}} \chi \varphi_{0 k} \mathrm{~d} x=\int_{\omega_{2}} \varphi_{0 k} \mathrm{~d} x$. Since $\varphi_{01}=\left|\Omega \backslash D_{0}\right|^{-\frac{1}{2}}$, and since by (4.4) $\int_{\omega_{2}} \varphi_{0 k} \mathrm{~d} x=0$, for all $k \geq 2$, it follows that $\chi=1$ in $\Omega \backslash \overline{D_{0}}$. This implies that $\omega_{2}=\Omega_{0} \backslash \overline{D_{0}}$, which leads to a contradiction. Then $D_{1}$ must be contained in $D_{0}$. By the same argument one can prove that $D_{0}$ must be contained in $D_{1}$, that is $D_{0}=D_{1}$.

The proof of Theorem 1.5 is complete.

Now we prove Theorem 1.6, that is the identifiability of $I$ and $D$ from the Neumann Boundary Spectral Data $\operatorname{NBSD}(I, D)$.

Proof of Theorem 1.6. Without loss of generality we can suppose that the boundaries $I_{j}, j \in\{0,1\}$, coincide, so $\Omega_{0}=\Omega_{1}=: \Omega$. We prove that $D_{0}=D_{1}$. Suppose, by contradiction, that $D_{1}$ is not contained in $D_{0}$. Let $D:=D_{0} \cup D_{1}$, and $\omega$ be the connected component of $\Omega \backslash \bar{D}$ such that $\partial \Omega \subset \partial \omega$. By hypothesis we know that the Neumann Boundary Spectral Data $\operatorname{NBSD}\left(I_{j}, D_{j}\right), j \in\{0,1\}$, coincide, i.e.

$$
\mu_{0 k}=\mu_{1 k}=: \mu_{k}, \quad \text { and } \quad \kappa \nabla \psi_{0 k} \cdot \mathbf{n}=\kappa \nabla \psi_{1 k} \cdot \mathbf{n} \quad \text { on } A \text {. }
$$

Putting $v_{k}:=\psi_{0 k}-\psi_{1 k}$, then $v_{k}$ solves:

$$
\left\{\begin{aligned}
-\operatorname{div}\left(\kappa \nabla v_{k}\right) & =\mu_{k} v_{k} & & \text { in } \omega, \\
v_{k} & =0 & & \text { on } \partial \Omega, \\
\kappa \nabla v_{k} \cdot \mathbf{n}=0 & =0 & & \text { on } A .
\end{aligned}\right.
$$

By the unique continuation principle, it follows that $v_{k} \equiv 0$ in $\bar{\omega}$, for all $k \geq 1$, i.e.

$$
\psi_{0 k}=\psi_{1 k} \quad \text { in } \bar{\omega}
$$


which implies

$$
\psi_{0 k}=\psi_{1 k} \quad \text { on } \partial \omega
$$

Now, since $\psi_{1 k}=0$ on $\partial D_{1} \cap \partial \omega$, equation (4.5) yields that the first eigenfunction $\psi_{01}=0$ on $\partial D_{1} \cap \partial \omega$, which leads to a contradiction since $\psi_{01}$ must be positive in $\Omega \backslash \overline{D_{0}}$ (see Courant and Hilbert [3], pp. 452-453). Then $D_{1}$ must be contained in $D_{0}$. By the same argument one can prove that $D_{0}$ must be contained in $D_{1}$, that is $D_{0}=D_{1}$.

The proof of Theorem 1.6 is complete.

\section{Concluding Remark}

Until now we have supposed that the known thermal diffusion coefficient $\kappa$ in (1.3) is independent of the time variable $t$. The aim of this section is to prove the identifiability of $I$ and $D$ in (1.3), in the case where the anisotropic diffusion coefficient $\kappa(t, x)$ depends also on the time variable $t$. Clearly the above boundary spectral data method does not apply anymore, because of the $t$ dependence of $\kappa$. In spite of that, under the hypothesis that $\kappa(t, x)$ is analytic in $t$, we can prove the following

Theorem 5.1. Under the hypothesis of Theorem 1.1, let $\kappa(t, x)$ be a symmetric $N \times N$ matrix-valued function in $[0, T] \times\left(\overline{\Omega_{0}} \cup \overline{\Omega_{1}}\right)$, satisfying assumption (1.1) in $[0, T] \times\left(\overline{\Omega_{0}} \cup \overline{\Omega_{1}}\right)$, such that $\kappa(\cdot, x)$ is analytic in $[0, T]$, for all $x \in \overline{\Omega_{0}} \cup \overline{\Omega_{1}}$, and $\kappa(t, \cdot) \in C^{1}\left(\overline{\Omega_{0}} \cup \overline{\Omega_{1}}\right)$ for all $t \in[0, T]$. Let $u_{j 0} \in H^{1}\left(\Omega_{j} \backslash D_{j}\right), \varphi_{j} \in C\left([0, T] ; L^{2}\left(\partial D_{j}\right)\right)$, $\psi_{j} \in C\left([0, T] ; L^{2}\left(I_{j}\right)\right), g \in C\left([0, T] ; L^{2}(A)\right)$ be such that $u_{j} \in C\left((0, T] ; H^{1}\left(\Omega_{j} \backslash D_{j}\right)\right) \cap C^{1}\left([0, T] ; L^{2}\left(\Omega_{j} \backslash D_{j}\right)\right)$ solve (1.3), when $\Omega:=\Omega_{j}, D:=D_{j}$, and $\kappa(x)$ is replaced by $\kappa(t, x)$. Suppose that, for a given time $T_{0} \in(0, T]$, and a fixed $\tau_{0}, 0<\tau_{0}<T_{0}$,

$$
u_{0}(t)_{\mid A_{\text {out }}}=u_{1}(t)_{\mid A_{\text {out }}} \quad \text { in } L^{2}\left(A_{\text {out }}\right)
$$

for $t \in\left[T_{0}-\tau_{0}, T_{0}\right]$, and for all $g \in C\left([0, T] ; L^{2}(A)\right)$ such that the $\operatorname{supp}(g(t, \cdot)) \subset A$ for $t \in[0, T]$. Then $I_{0}=I_{1}$ and $D_{0}=D_{1}$.

We obseve that in Theorem 5.1 the thermic diffusion coefficient $\kappa(t, \cdot) \in C^{1}\left(\overline{\Omega_{0}} \cup \overline{\Omega_{1}}\right)$, and that the temperatures $u_{j}(t)_{\mid A_{\text {out }}}$ coincide in the whole interval of time $\left[T_{0}-\tau_{0}, T_{0}\right]$.

We premise the proof of Theorem 5.1 with the following:

Lemma 5.2. Under the hypothesis of Theorem 5.1, we have

$$
u_{0}=u_{1} \quad \text { in }\left[0, T_{0}-\tau_{0}\right] \times\left(\left(\Omega_{0} \backslash \overline{D_{0}}\right) \cap\left(\Omega_{1} \backslash \overline{D_{1}}\right)\right)
$$

for all $g$ as in Theorem 5.1.

Once this result is at hand we can prove Theorem 5.1 .

Proof of Theorem 5.1. We divide the proof into two steps. In the first step we prove that $I_{0}=I_{1}$, in the second that $D_{0}=D_{1}$.

Step 1. As usual (see Lem. 3.3) we reduce to the case where the data $u_{j 0}, \varphi_{j}, \psi_{j}$ are identically zero. By contradiction suppose that $I_{0} \neq I_{1}$. By Lemma 5.2 we know that

$$
u_{0}=u_{1} \quad \text { in }\left[0, T_{0}-\tau_{0}\right] \times \omega_{0},
$$

where $\omega_{0}:=\left(\Omega_{0} \backslash \overline{D_{0}}\right) \cap\left(\Omega_{1} \backslash \overline{D_{1}}\right)$. In particular $(5.7)$ yields

$$
\kappa \nabla u_{0} \cdot \mathbf{n}=\kappa \nabla u_{1} \cdot \mathbf{n} \quad \text { on }\left[0, T_{0}-\tau_{0}\right] \times \partial \omega_{0} .
$$


Let us denote by

$$
\Omega:=\overline{\Omega_{0}} \cup \overline{\Omega_{1}}
$$

and

$$
\omega_{1}:=\Omega \backslash \overline{\Omega_{1}} .
$$

Multiplying $u_{0}$ by $u_{0}$, and integrating over $\left(0, t_{0}\right) \times \omega_{1}$, where $0<t_{0} \leq T_{0}-\tau_{0}$, we obtain

$$
\int_{\omega_{1}} \int_{0}^{t_{0}} \partial_{t} u_{0} u_{0} \mathrm{~d} t \mathrm{~d} x-\int_{\omega_{1}} \int_{0}^{t_{0}} \operatorname{div}\left(\kappa(t, x) \nabla u_{0}\right) \nabla u_{0} \mathrm{~d} t \mathrm{~d} x=0 .
$$

Hence integrating by parts the second term over $\omega_{1}$, using $(5.8)$, and since $\kappa \nabla u_{j} \cdot \mathbf{n}=0$ on $(0, T) \times I_{j}$, we have

$$
\begin{aligned}
0 & =\int_{\omega_{1}} \int_{0}^{t_{0}} \partial_{t} u_{0} u_{0} \mathrm{~d} t \mathrm{~d} x+\int_{\omega_{1}} \int_{0}^{t_{0}} \kappa(t, x) \nabla u_{0} \cdot \nabla u_{0} \mathrm{~d} t \mathrm{~d} x \\
& \geq \frac{1}{2} \int_{\omega_{1}} \int_{0}^{t_{0}} \partial_{t}\left|u_{0}\right|^{2} \mathrm{~d} t \mathrm{~d} x+\alpha \int_{\omega_{1}} \int_{0}^{t_{0}}\left|\nabla u_{0}\right|^{2} \mathrm{~d} t \mathrm{~d} x \\
& =\frac{1}{2} \int_{\omega_{1}}\left|u_{0}\left(t_{0}\right)\right|^{2} \mathrm{~d} x+\alpha \int_{\omega_{1}}^{t_{0}}\left|\nabla u_{0}\right|^{2} \mathrm{~d} t \mathrm{~d} x,
\end{aligned}
$$

where the first step is obtained recalling that $\kappa(t, x) \xi \cdot \xi \geq \alpha$, for a constant $\alpha>0$. This implies

$$
0 \geq \frac{1}{2} \int_{\omega_{1}}\left|u_{0}\left(t_{0}\right)\right|^{2} \mathrm{~d} x+\alpha \int_{\omega_{1}} \int_{0}^{t_{0}}\left|\nabla u_{0}\right|^{2} \mathrm{~d} t \mathrm{~d} x
$$

which leads to a contradiction.

Step 2. In this step we prove that $D_{0}=D_{1}$. First we prove that $D_{1} \subseteq D_{0}$. By contradiction we suppose that $D_{1}$ is not contained in $D_{0}$. By the previous step $I_{0}=I_{1}$, so $\Omega_{0}=\Omega_{1}:=\Omega$. Let $D:=D_{0} \cup D_{1}$, and $\omega$ be the connected component of $\Omega \backslash \bar{D}$ such that $\partial \Omega \subset \partial \omega$. Now let us denote by

$$
\omega_{2}:=(\Omega \backslash \omega) \backslash \overline{D_{0}}
$$

Multiplying the solution $u_{0}$ of $(1.3)$ by $u_{0}$, and integrating over $\left(0, t_{0}\right) \times \omega_{2}$, where $0<t_{0} \leq T_{0}-\tau_{0}$, we obtain

$$
\int_{\omega_{2}} \int_{0}^{t_{0}} \partial_{t} u_{0} u_{0} \mathrm{~d} t \mathrm{~d} x-\int_{\omega_{2}} \int_{0}^{t_{0}} \operatorname{div}\left(\kappa(t, x) \nabla u_{0}\right) \nabla u_{0} \mathrm{~d} t \mathrm{~d} x=0 .
$$


Hence integrating by parts over $\omega_{2}$, using (5.8), and since $\kappa \nabla u_{j} \cdot \mathbf{n}=0$ on $(0, T) \times\left((\partial \Omega \backslash A) \cup \partial D_{j}\right)$, we have

$$
\begin{aligned}
0 & =\int_{\omega_{2}} \int_{0}^{t_{0}} \partial_{t} u_{0} u_{0} \mathrm{~d} t \mathrm{~d} x+\int_{\omega_{2}} \int_{0}^{t_{0}} \kappa(t, x) \nabla u_{0} \cdot \nabla u_{0} \mathrm{~d} t \mathrm{~d} x \\
& \geq \frac{1}{2} \int_{\omega_{2}}\left|u_{0}\left(t_{0}\right)\right|^{2} \mathrm{~d} x+\alpha \int_{\omega_{2}} \int_{0}^{t_{0}}\left|\nabla u_{0}\right|^{2} \mathrm{~d} t \mathrm{~d} x,
\end{aligned}
$$

which leads to a contradiction. Then $D_{1}$ must be contained in $D_{0}$. By the same argument one can prove that $D_{0}$ must be contained in $D_{1}$, that is $D_{0}=D_{1}$.

The proof of Theorem 5.1 is complete.

Now we prove Lemma 5.2.

Proof of Lemma 5.2. We divide the proof into three steps.

Step 1. Let us denote by $u:=u_{0}-u_{1}$ in $(0, T) \times \omega_{0}$ (we recall that the data $u_{j 0}, \varphi_{j}, \psi_{j}$ are identically zero). Then $u$ solves:

$$
\left\{\begin{array}{rlll}
\partial_{t} u-\operatorname{div}(\kappa(t, x) \nabla u) & = & & \text { in }(0, T) \times \omega_{0} \\
u(0) & = & 0 & \text { in } \omega_{0}, \\
\kappa \nabla u \cdot \mathbf{n} & = & 0 & \text { on }(0, T) \times A
\end{array}\right.
$$

where $A:=\left(\partial \Omega_{j}\right) \backslash I_{j}$. By hypothesis $u=0$ on $\left[T_{0}-\tau_{0}, T_{0}\right] \times A_{\text {out }}$. So, by the unique continuation principle (see Saut and Scheurer [7]), it follows that $u \equiv 0$ in $\left[T_{0}-\tau_{0}, T_{0}\right] \times \omega_{0}$, that is

$$
u_{0} \equiv u_{1} \quad \text { in }\left[T_{0}-\tau_{0}, T_{0}\right] \times \omega_{0}
$$

for all $g \in C\left([0, T] ; L^{2}(A)\right)$ such that the support $\operatorname{supp}(g(t, \cdot)) \subset A$ for $t \in[0, T]$.

Step 2. Let $G_{j}(t, \tau ; x, y)$ be the Green functions related to problem (1.3) when $\Omega:=\Omega_{j}, D:=D_{j}$, and $\kappa(x)$ is replaced by $\kappa(t, x)$, that is

$$
\left\{\begin{array}{rlrl}
\partial_{t} G_{j}(t, \tau ; x, y)-\operatorname{div}\left(\kappa(t, x) \nabla G_{j}(t, \tau ; x, y)\right) & =0 & & \text { in }(\tau, T) \times \Omega_{j} \backslash \overline{D_{j}}, \\
G_{j}(\tau, \tau ; x, y) & =\delta_{y} & \text { in } \Omega_{j} \backslash \overline{D_{j}}, \\
\kappa \nabla G_{j}(t, \tau ; \sigma, y) \cdot \mathbf{n} & =0 & \text { on }(\tau, T) \times \partial D_{j}, \\
\kappa \nabla G_{j}(t, \tau ; \sigma, y) \cdot \mathbf{n} & =0 & & \text { on }(\tau, T) \times \partial \Omega_{j},
\end{array}\right.
$$

where $0 \leq \tau<T, y \in \Omega_{j} \backslash \overline{D_{j}}$, and $\delta_{y}$ is the delta Dirac with pole in $y$. Let us note that, since the coefficient $\kappa(t, x)$ is analytic in $t$, the Green functions $\kappa_{j}(t, \tau ; x, y)$ are analytic in the variables $t, \tau$ for $t>\tau$. Moreover (see for example Ladyzhenskaja et al. [5], p. 408) the solutions $u_{j}$ can be written in the following form:

$$
u_{j}(t, y)=\int_{0}^{t} \int_{A} G_{j}\left(t, \tau ; \sigma^{\prime}, y\right) g\left(\tau, \sigma^{\prime}\right) \mathrm{d} \sigma^{\prime} \mathrm{d} \tau
$$

for $(t, y) \in[0, T) \times \Omega_{j} \backslash \overline{D_{j}}$. Choosing $g$ such that $g \equiv 0$ on $\left[T_{0}-\varepsilon_{0}, T_{0}\right] \times A$, where $\tau_{0}<\varepsilon_{0}<T_{0}$, we have

$$
u_{j}(t, y)=\int_{0}^{T_{0}-\varepsilon_{0}} \int_{A} G_{j}\left(t, \tau ; \sigma^{\prime}, y\right) g\left(\tau, \sigma^{\prime}\right) \mathrm{d} \sigma^{\prime} \mathrm{d} \tau,
$$


for $(t, y) \in\left[T_{0}-\tau_{0}, T_{0}\right] \times \Omega_{j} \backslash \overline{D_{j}}$. So, from (5.11), and using (5.13), it follows that

$$
\int_{0}^{T_{0}-\varepsilon_{0}} \int_{A}\left(G_{0}\left(t, \tau ; \sigma^{\prime}, y\right)-G_{1}\left(t, \tau ; \sigma^{\prime}, y\right)\right) g\left(\tau, \sigma^{\prime}\right) \mathrm{d} \sigma^{\prime} \mathrm{d} \tau=0
$$

for $(t, y) \in\left[T_{0}-\tau_{0}, T_{0}\right] \times \omega_{0}$. Assuming that $g\left(\tau, \sigma^{\prime}\right) \equiv 0$ for $\tau \neq\left[T^{\prime}-\varepsilon^{\prime}, T^{\prime}+\varepsilon^{\prime}\right]$, and $\sigma^{\prime} \in A$, where $T^{\prime}$ is a fixed time, $T^{\prime} \in\left(0, T_{0}-\varepsilon_{0}\right)$, and $0<\varepsilon^{\prime}<T^{\prime}$, (5.14) becomes

$$
\int_{T^{\prime}-\varepsilon^{\prime}}^{T^{\prime}+\varepsilon^{\prime}} \int_{A}\left(G_{0}\left(t, \tau ; \sigma^{\prime}, y\right)-G_{1}\left(t, \tau ; \sigma^{\prime}, y\right)\right) g\left(\tau, \sigma^{\prime}\right) \mathrm{d} \sigma^{\prime} \mathrm{d} \tau=0,
$$

for all such functions $g$. Hence we have

$$
G_{0}\left(t, \tau ; \sigma^{\prime}, y\right)=G_{1}\left(t, \tau ; \sigma^{\prime}, y\right)
$$

in $\left[T_{0}-\tau_{0}, T_{0}\right] \times\left[T^{\prime}-\varepsilon^{\prime}, T^{\prime}+\varepsilon^{\prime}\right] \times A \times \omega_{0}$.

Step 3. In this step we prove the assertion of the lemma. For a fixed $t \in\left[T_{0}-\tau_{0}, T_{0}\right]$, since the Green functions $G_{j}\left(t, \tau ; \sigma^{\prime}, y\right)$ are analytic in $\tau$ for $\tau<t$, the unique continuation principle of analytic functions of the variable $\tau$ implies that (5.16) holds for $\tau \in[0, t)$. So (5.16) holds in

$$
\left[T_{0}-\tau_{0}, T_{0}\right] \times\left[0, T_{0}-\tau_{0}\right) \times A \times \omega_{0} .
$$

Conversely, for a fixed $\tau \in\left[0, T_{0}-\tau_{0}\right)$, since the Green functions $G_{j}\left(t, \tau ; \sigma^{\prime}, y\right)$ are analytic in $t$ for $t>\tau$, by the unique continuation principle of analytic functions of the variable $t(5.16)$ holds for $t \in\left(\tau, T_{0}\right]$. So, from (5.12), we conclude that $u_{0}(t, y)=u_{1}(t, y)$ in $\left[0, T_{0}-\tau_{0}\right] \times \omega_{0}$, for all $g \in C\left([0, T] ; L^{2}(A)\right)$ such that the $\operatorname{support} \operatorname{supp}(g(t, \cdot)) \subset A$ for $t \in[0, T]$.

The proof of Lemma 5.2 is complete.

The author is grateful to Prof. Otared Kavian and Prof. Sergio Vessella for useful discussions and very helpful suggestions of the results.

\section{REFERENCES}

[1] K. Bryan and L.F. Caudill, An Inverse Problem in Thermal Imaging. SIAM J. Appl. Math. 56 (1996) 715-735.

[2] B. Canuto and O. Kavian, Determining Coefficients in a Class of Heat Equations via Boundary Measurements. SIAM J. Math. Anal. (to appear).

[3] R. Courant and D. Hilbert, Methods of Mathematical Physics, Vol. 1. Wiley, New York (1953).

[4] N. Garofalo and F.H. Lin, Monotonicity Properties of Variational Integrals, $A_{p}$ Weights and Unique Continuation. Indiana Univ. Math. J. 35 (1986) 245-268.

[5] O.A. Ladyzhenskaja, V.A. Solonnikov and N.N. Uralceva, Linear and Quasilinear Equations of Parabolic Type. AMS, Providence, RI, Trans. Math. Monographs 23 (1968).

[6] Rakesh and W.W. Symes, Uniqueness for an Inverse Problem for the Wave Equation. Comm. Partial Differential Equations 13 (1988) 87-96.

[7] J.-C. Saut and B. Scheurer, Unique Continuation for Some Evolution Equations. J. Differential Equations 66 (1987) $118-139$.

[8] S. Vessella, Stability Estimates in an Inverse Problem for a Three-Dimensional Heat Equation. SIAM J. Math. Anal. 28 (1997) 1354-1370.

[9] S. Vessella, Private Comunication. 